\title{
UAV Path Planning Optimization based on GNSS Quality and Mission Requirements
}

\author{
Nikolaos Nanos ${ }^{1}$, Oguz Kagan Isik ${ }^{2}$, Ivan Petrunin ${ }^{3}$, Dimitrios \\ Panagiotakopoulos ${ }^{4}$, Antonios Tsourdos ${ }^{5}$ \\ Cranfield University, Bedfordshire, MK43 OAL, United Kingdom \\ Ricardo Verdeguer Moreno ${ }^{6}$
}

Spirent Communications PLC, West Sussex, RH10 1BD, United Kingdom

One of the most crucial factors for the overall success of an Unmanned Aerial Vehicle (UAV) mission is navigation performance, which is severely affected in Global Navigation Satellite Systems (GNSS) challenging environments. A solution to this problem could come through path planning optimization. This paper investigates the impact that GNSS quality information included in the UAV path planning process would have on the overall UAV mission success rate (MSR) when flying through an urban canyon. Number of visible satellites and Horizontal Dilution of Precision (HDOP) in addition to mission-specific requirements are given as input to the Particle Swarm Optimization (PSO) algorithm to calculate the optimal path for two cases. One includes the GNSS observables, and the other does not. Optimal paths for three different altitudes are obtained. All paths are simulated by a GNSS signal simulator, including a comprehensive multipath model. GNSS data are collected by a hardware receiver for analysis of the UAV positioning error and GNSS availability. Mission failures cases are defined accordingly, and the overall mission success rate (MSR) of each scenario is assessed. By analyzing the findings, it is concluded that in $83 \%$ of cases, the path planning process that included GNSS information was able to increase the MSR. Also, the increase in MSR was bigger when flying at low altitude.

\section{Nomenclature}

$\begin{array}{ll}\text { AL } & =\text { Alert Limit } \\ \text { BVLOS } & =\text { Beyond Visual Line of Sight } \\ \text { CAA } & =\text { Civil Aviation Authority } \\ \text { FAA } & =\text { Federal Aviation Administration } \\ f_{\text {cost }} & =\text { Cost Function } \\ \text { GNSS } & =\text { Global Navigation Satellite System } \\ \text { GPS } & =\text { Global Positioning System } \\ \text { HAL } & =\text { Horizontal Alert Limit } \\ \text { HDOP } & =\text { Horizontal Dilution of Precision } \\ \text { ICAO } & =\text { International Civil Aviation Organization } \\ \text { MF } & =\text { Mission Failure }\end{array}$

${ }^{1}$ Graduate student, School of Aerospace, Transport and Manufacturing (SATM), Cranfield University

${ }^{2} \mathrm{PhD}$ candidate, School of Aerospace, Transport and Manufacturing (SATM), Cranfield University

${ }^{3}$ Lecturer, Centre for Autonomous and Cyber-physical Systems, Cranfield University

${ }^{4}$ Senior lecturer, Centre for Autonomous and Cyber-physical Systems, Cranfield University

${ }^{5}$ Professor, Centre for Autonomous and Cyber-physical Systems, Cranfield University

${ }^{6}$ Product manager for Connected Autonomous Vehicle Applications, Spirent Communications PLC 


$\begin{array}{ll}\text { MSR } & =\text { Mission Success Rate } \\ \text {N SIM }_{\text {NVS }} & =\text { Number of Simulations } \\ \text { SVID } & =\text { Spamber of Visible Satellites } \\ \text { TTA } & =\text { Time-to-Alert } \\ \text { UAM } & =\text { Urban Air Mobility } \\ \text { UAV } & =\text { Unmanned Aerial Vehicle } \\ \text { UTM } & =\text { UAV Traffic Management }\end{array}$

\section{II.Introduction}

The use of UAV is of increasing popularity nowadays for civilian, military and commercial operations around the world. There are multiple factors that can contribute to an overall successful autonomous UAV mission. One of the most critical is navigation performance, as most UAV missions rely exclusively on the onboard GNSS receiver for navigating correctly and accurately. This is typically sufficient for missions that are operated in open sky environments. However, in dense urban environments, it is widely common for the GNSS signals to be severely degraded or even completely missing. Therefore, in order to ensure successful future Urban Air Mobility (UAM) missions, various techniques have been investigated and proposed that enhance the performance in urban environments with degraded GNSS quality.

One of these techniques is path planning optimization, which has been identified as a good way to de-risk operations by taking into consideration various mission parameters and produce a flight path that would minimize their impact on the mission. A lot of these mission parameters have been identified in the literature. For instance, [1] considered the total UAV flight distance, the maximum speed, time, maximum weight and minimum battery depth of discharge in their work. Additionally, [2] describe the 'no-fly zones' that a UAV cannot enter during its flight. These are defined by the National Aviation Authorities (like Civil Aviation Authority (CAA) in the UK or Federal Aviation Administration (FAA) in the USA) and include airspace over sensitive facilities like airports, national parks, prisons and more. Also, regarding safety, [3] highlights the importance of keeping the existing minimum separation requirements. These can be either for another UAV flying in the area or obstacles such as buildings.

It is obvious that the choice for which of the above requirements needs to be considered during the path planning process depends on the UAV mission scenario itself. Taking all these into consideration, in our paper we focus on investigating what the benefits of including GNSS quality information in the UAV path planning process would be and how the benefits can be evaluated in terms of UAV MSR.

More specifically, in order to address this problem, a UAV path planning optimization process is developed, which takes into consideration the GNSS quality and other constraints of a specific UAV mission scenario and produces an optimal trajectory for the given parameters. Multiple trajectories are produced by the optimization process for various cases, which are then analyzed using GNSS tools, and data from a GNSS receiver are used to evaluate its MSR.

For the assessment of the MSR of the case scenarios, first, the mission failure had to be defined. The idea was to follow the guidance of [4] where it states that for Beyond Visual Line of Sight (BVLOS) UAV flights, there should be an operational volume defined for the flight to be conducted safely inside its boundaries and an emergency buffer surrounding the operational volume, as depicted in Figure 1.

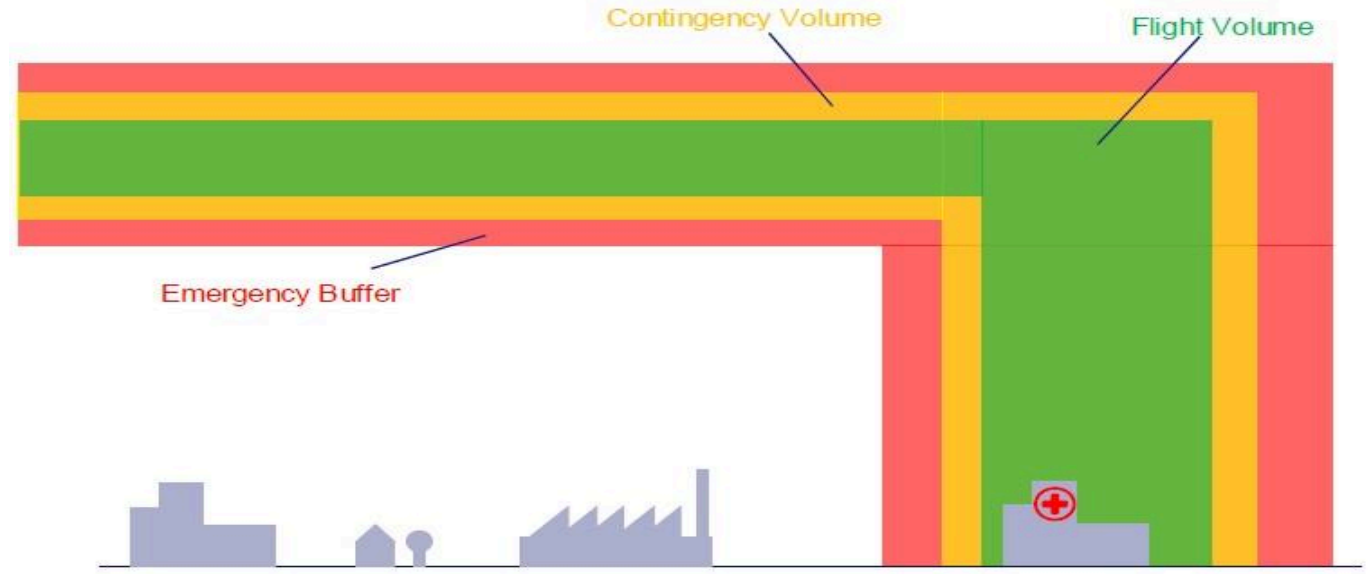

Figure 1 Operational volume and emergency buffer according to CAA CAP1915 [4] 
The operational volume consists of the flight volume and the contingency volume. Flight volume is the area inside which the entire mission should be operated. It includes a sufficient buffer available for all flight movements and maneuvers, as well as navigational errors and weather conditions. The contingency volume provides extra space around the flight volume so that if the UAV enters inside it, predefined procedures should be triggered so that it enters back into the flight volume. If it fails to do so and enters the emergency buffer, then emergency procedures should be triggered for safely flight termination.

In order to solve the UAV path planning problem, researchers have used various techniques throughout the years. The recent work of [5] has presented a complete review of the available techniques for path planning and categorized them in representation techniques, cooperative techniques, non-cooperative techniques, and coverage and connectivity. In recent studies, there is a trend to use bio-inspired models for path planning. This is done because, in the case of complex multi-objective problems, other techniques struggle to produce an optimal solution to the path planning problem. Instead, bio-inspired techniques manage to converge to the desired goal. One popular optimization algorithm of this category is Particle Swarm Optimization (PSO). It is widely used in various optimization problems because of its simple implementation and can converge very quickly. It has only a few parameters for tuning, which makes it more insensitive compared to other algorithms. Also, variants of the original algorithm from [6] have been proposed in the literature, which makes the algorithm even more efficient and solve some of its issues like premature convergence and local minima trap.

For including navigation performance into the path planning process, the performance criteria should be determined initially. The common criteria of GNSS performance, which are accepted and used in this paper, are defined by ICAO [7] with integrity and availability terms as below:

- Integrity: The measure of trust that can be placed in the correctness of the information supplied by a navigation system.

- Availability: The percentage of time that the services of the system are usable by the navigator.

On the research area of UAV path planning by considering GNSS performance, work [8] developed a pre-flight planning method in an urban environment where the GNSS availability is assessed in order to prove the necessity of using alternative navigation sensors. Their approach was to create a tool that will generate heatmaps of visible satellites and Dilution of Precision (DOP) values, which then are used by an optimization algorithm to produce the optimal path. Their model included only information of loss of direct line of sight and they suggested for future work to enhance it with better models of multipath, scattering and reflections. Our intention in this paper is to fill this gap by including a realistic multipath model, which would simulate diffractions and reflections, and thus to effectively assess their impact on GNSS signal quality, path planning, as well as GNSS availability and integrity metrics.

In their work, authors of study [9] created a tool that would model the multipath effect in an urban area in Hong Kong, by using 3D building models and ray-tracing simulations. Then, they used this tool to predict the GPS positioning error in different heights and put it in an error map which then is used by $\mathrm{A}^{*}$ optimization algorithm to create an optimal flight path. They verify the predicted model by experimental data of UAV carried by pedestrians. They highlighted as a weak point the fact that the error map creation demanded high computational efforts. Also, the output of the optimization algorithm produced sharp turning angles, which may not allow flights for all UAV platforms. Therefore, in our approach, one of the constraints included in the path planning optimization process is the maximal heading angle change of the UAV, so that the generated path is as realistic as possible.

Authors of work [10] propose a path planning strategy for a swarm of UAVs in an environment with mixed GNSS coverage. They aim to show that with cooperation techniques such as the use of father-son approach where the father UAV is outside the GNSS challenging environment, the GNSS challenging areas can be passed with safety by the son UAV. In order to define the GNSS challenging areas, they use a threshold for accepted DOP value. They verified the results with real-time experiments. They conclude that the use of multiple constellations (GPS, GLONASS, Galileo) provided better results as the number of challenging areas with high DOP was reduced significantly in this case. In our research method, we included not only the DOP values for characterizing the GNSS quality but also information for the number of visible satellites (NVS).

Taking all the above into consideration, the overall aim of our paper is to investigate the possible impact on a UAV mission success rate, if GNSS quality parameters would be included in the UAV path planning process. The novel contribution of this work is in embedding of multiple GNSS quality metrics (DOP and number of visible satellites) into UAV path planning process with comprehensive analysis of multipath effect contribution, definition and use of mission success rate for quality analysis of the generated UAV trajectory.

The rest of the paper is organized as follows. Section III contains a detailed description of our methodology to produce the desired results, the rationale behind chosen approaches and the research limitations. In Section IV, the results are analyzed and discussed. Finally, Section V contains the conclusions of this paper. 


\section{III.Simulations and Results}

\section{A. Simulation Setup}

In order to determine the impact that the GNSS signal quality has in the path planning process and the mission success itself, two cases are considered in this research. In the first case, the path planning procedure would include all other mission requirements except for the GNSS signal quality, which would be added only in the second case. By comparing the results obtained by the two cases, important findings could be extracted regarding the impact of GNSS of the final trajectory and subsequent mission success.

The most realistic results could be obtained if the case studies would run in a real environment using a UAV with an onboard GNSS receiver to take the necessary measurements. However, due to obvious difficulties that this approach would have had, we decided to go for an alternative approach which included both hardware and software solutions provided by Spirent Communications plc, so that the above case studies could be simulated in the university lab. The tools used were the following:

- The GSS7000 GNSS simulator [11], which is capable of reproducing accurate and reliable multi-frequency, multi-GNSS RF signals and can be connected to an GNSS receiver through its RF output.

- The SimGEN software [12] which is responsible for operating the GSS7000. In SimGEN various scenario parameters can be defined, and the UAV trajectories can be simulated.

- The Sim3D software [13] which is a realistic multipath and obscuration simulation software. It connects with SimGEN and GSS7000 and reproduces the impact that a local environment may have on the GNSS signals (multipath effect).

- The employed GNSS receiver was Ublox NEO-M8N [14] connected to a serial COM port of the PC running SimGEN (baud rate 9600). The GNSS data collection frequency was set to be $1 \mathrm{~Hz}$.

For each scenario simulation, the GNSS data captured by the GNSS receiver were recorded for processing. These data were in NMEA file format, which is a standard format for the majority of GNSS receivers. The NMEA file consists of multiple sentences. Each sentence starts with a specific word called "data type" which defines the content of the sentence. The data type used for analysis in this paper is the "GGA" data type, which provides time, position and fix related information.

The positioning information which are in the dm (degrees minutes) format in the GGA sentence, need to be translated to the ENU (East North Up) coordinate system with reference point the starting point of every simulation.

The Hardware in the Loop (HIL) simulation setup that was used can be seen in Figure 2.

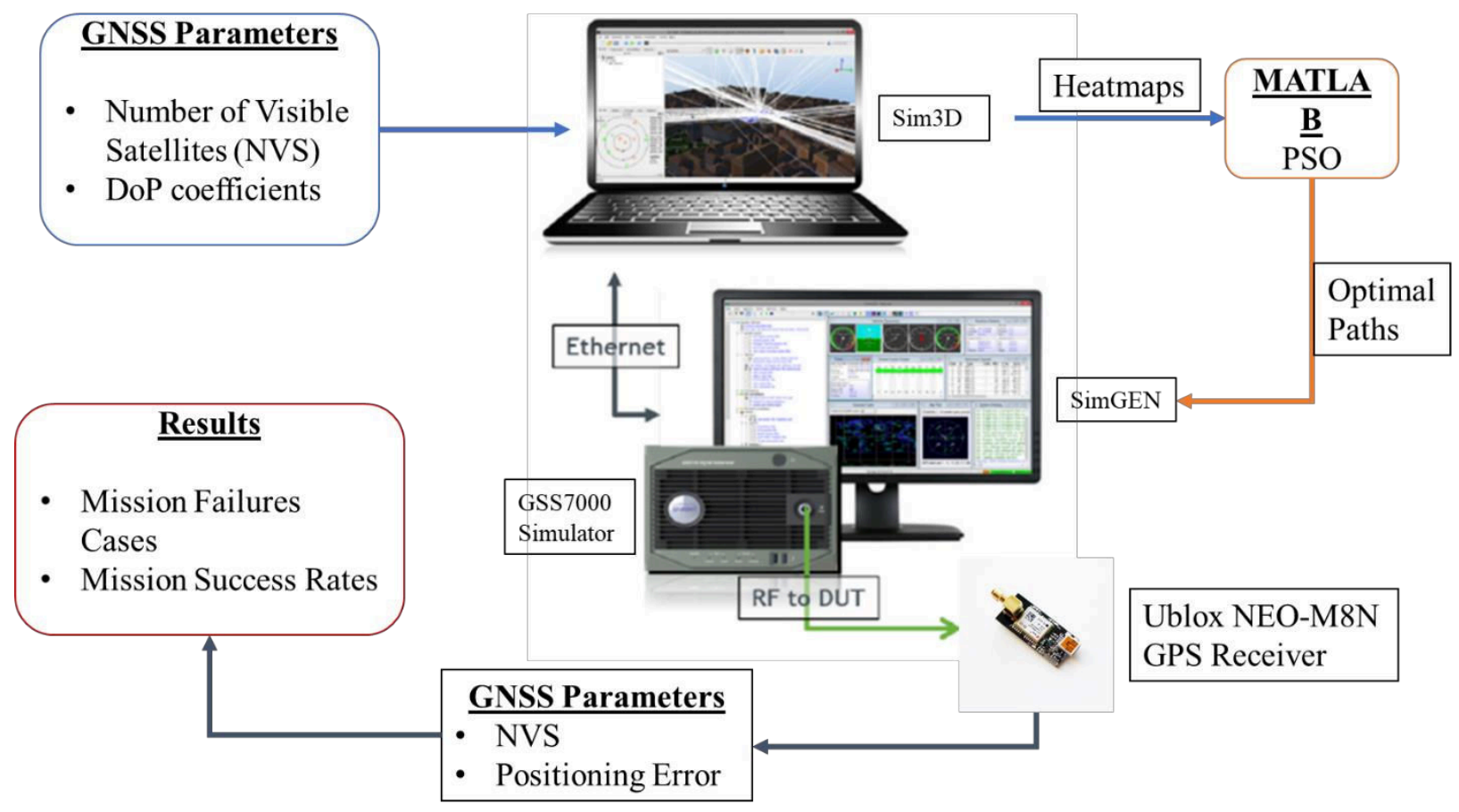

Figure 2 Simulation setup 
Sim3D's realistic 3D model of downtown Miami environment (buildings, streets, objects) was chosen to be the UAV mission scenario environment, as it includes an urban canyon region as well as suburban areas where we could investigate the impact of GNSS signal in the path planning performance and the corresponding mission success rate.

One of the capabilities of the Sim3D software is that it can generate GNSS performance heatmaps for various GNSS parameters. These were used in the next stage of the research method as an input in the path planning optimization algorithm to compute the optimal trajectory of the UAV mission.

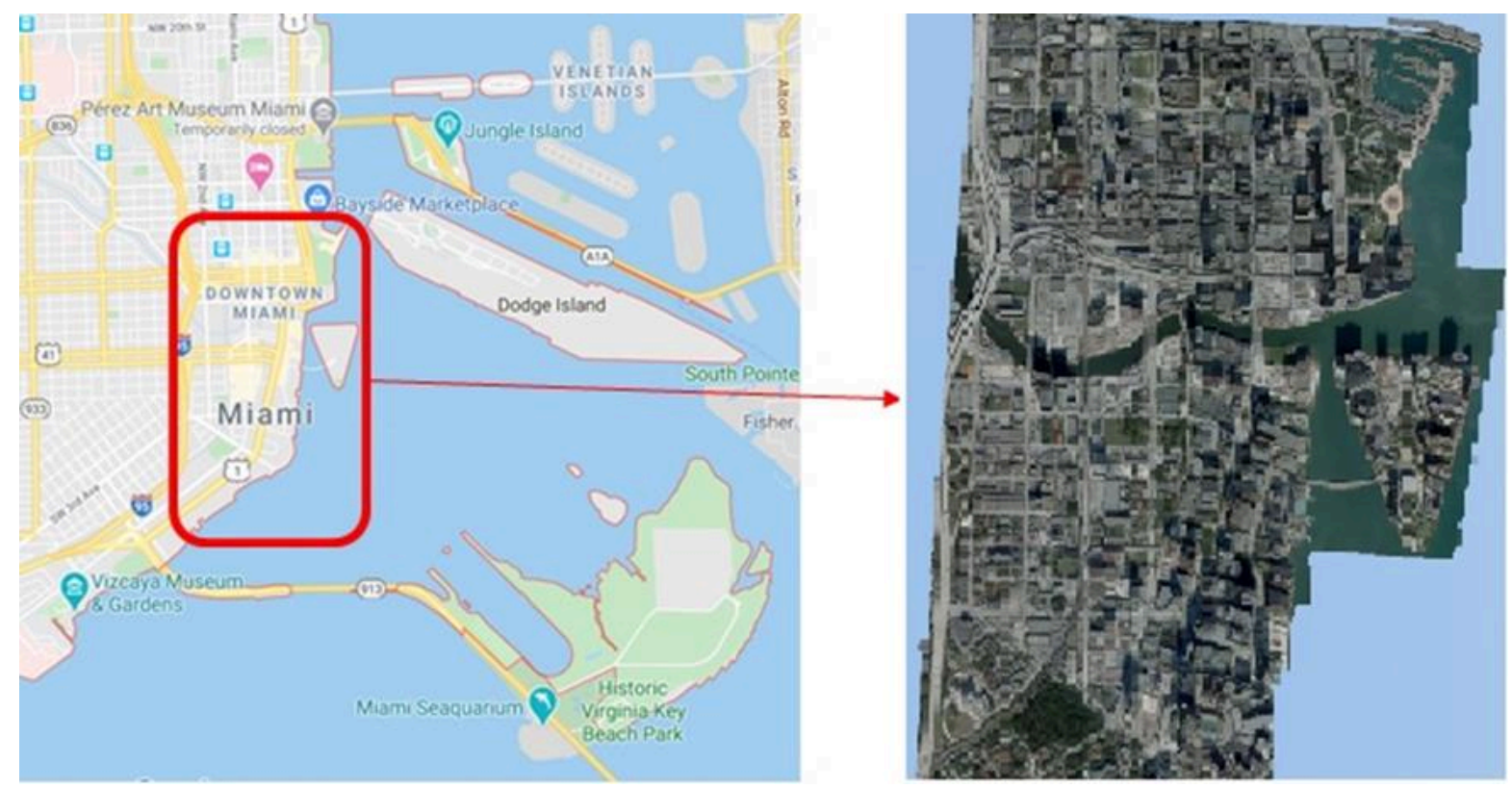

Figure 3 Mission environment (Miami Downtown) (https://www.google.com/maps)

\section{B. Multipath}

For the multipath effect of the environment being applied into the simulation, the Sim3D software had to be connected with the SimGEN software to run in parallel. The options that were available to be changed in the Sim3D were the following:

- Date/Time of simulation.

- Flight Altitude.

- Number of multipath traces per Space Vehicle ID (SVID), which relates to the number of GNSS signal beams originated from the same satellite that would be taken into consideration for the multipath calculation.

- Maximum number of reflections of the selected environment (e.g. buildings, ground, and objects) that a GNSS signal beam could have before reaching the GNSS receiver onboard the UAV.

The last two parameters can be better understood by looking at Figure 4. The number of multipaths per SVID option was set at 0,1 and 2 based on hardware capabilities of the GSS7000 simulator. The number of reflections was set to either 1 or 2 because after two reflections, the signal attenuation was found to be too high to make an effect in the GNSS receiver.

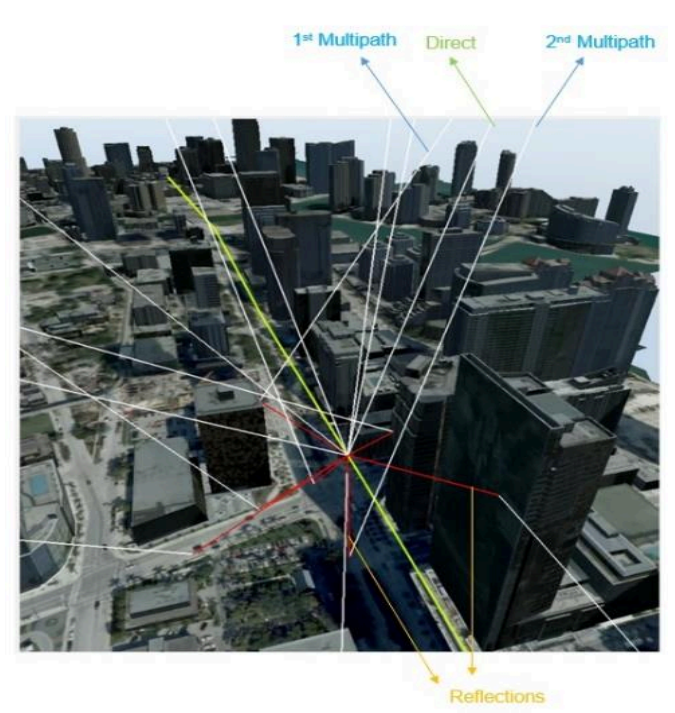

Figure 4 Multipath effect simulation

\section{Heatmap Generation}

For the selected Miami case scenario, it was decided to produce and use the following heatmaps.

- Building heatmap

- Number of visible satellites heatmap

- HDOP heatmap 
The building heatmap will be used for the first simulation scenario, where GNSS quality would not be considered in the path planning procedure. The other two heatmaps will be used in the second simulation scenario, which includes the impact of GNSS performance.

The heatmaps were generated for three specific flight altitudes: $10 \mathrm{~m}, 30 \mathrm{~m}$ and $100 \mathrm{~m}$ above ground. The altitude selection was made in compliance with the CAA Air Navigation Order (ANO) 2016, which defines the maximum flight altitude for a Small Unmanned Aircraft (SUA), having a weight of less than $20 \mathrm{~kg}$, to be $120 \mathrm{~m}$ (400 ft) [15]. That is also in-line with FAA Unmanned Traffic Management (UTM) concept of operations [16].

The building heatmaps generated by the Sim3D software do not change as the altitude increases; therefore, it was not possible to account for an increase of flight volume availability (building-free) with altitude change. So, regardless of the altitude level, we assumed that the buildings in the surrounding environment are tall enough to be considered as obstructions in flight path optimization. For the heatmaps concerning the number of visible satellites and HDOP, appropriate thresholds were used, considering the findings in [17]. As a result, the path planning algorithm would penalize use of the areas where these thresholds (NVS $<4$ and HDOP $>3$ ) are exceeded. Illustration of two types of heatmaps can be seen in Figure 5.

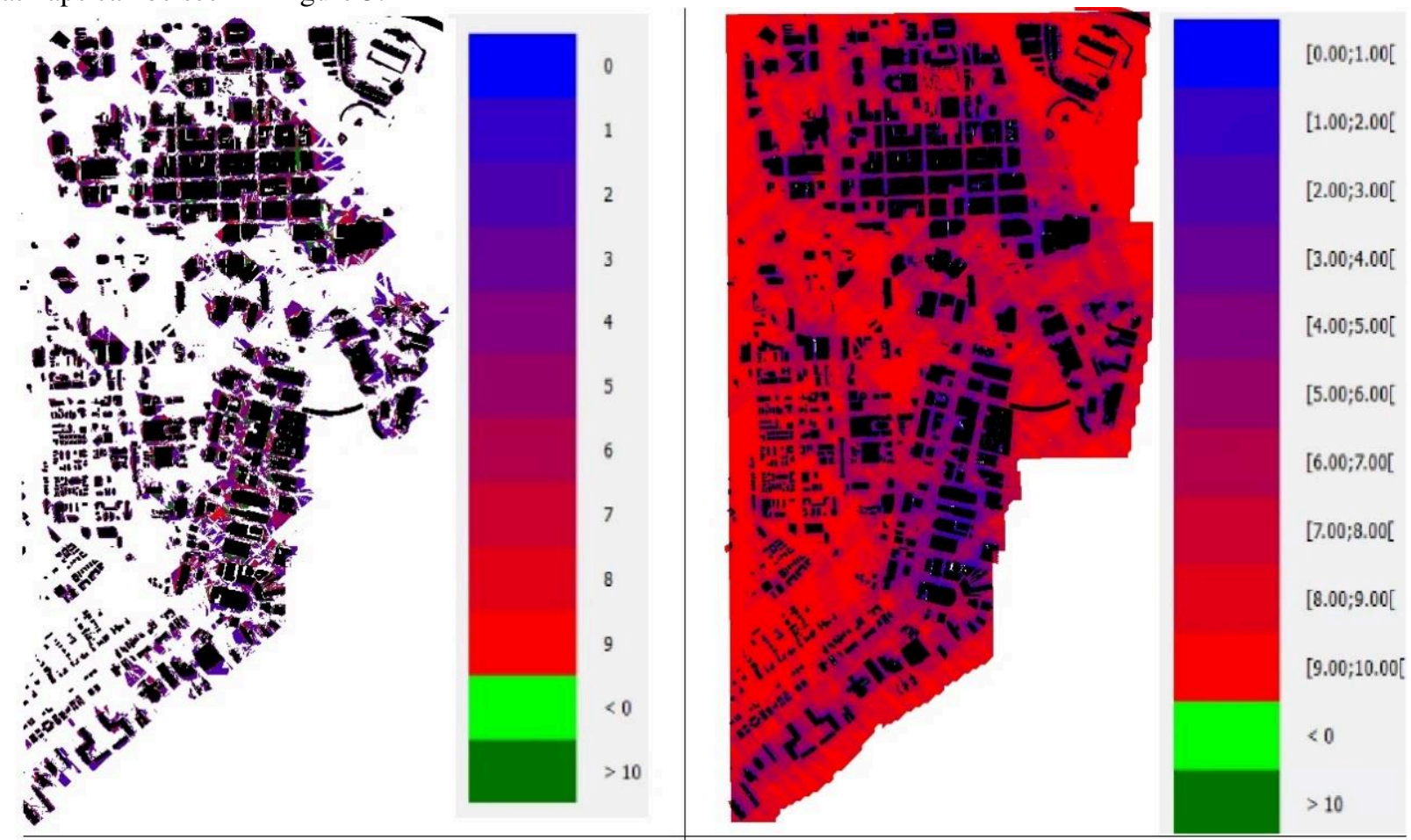

Figure 5 Miami heatmaps: NVS (Left) - HDOP (Right)

\section{Path Planning}

For the path planning (optimization) process, the PSO algorithm was chosen for its simple concept and ease of implementation, as well as its popularity and efficacy in similar optimization problems.

The aim of the path planning algorithm is to produce an optimal flight trajectory for each case by including in its computation the following mission requirements:

- Flight distance minimization

- Obstacle avoidance (buildings)

- Maneuverability constraints (change of heading angle)

- Avoidance of areas with low GNSS quality (for the $2^{\text {nd }}$ case study)

The choice of the above parameters was made under the hypothetical scenario that the mission would simulate an emergency medical transportation, so the most important aspect of being considered was time, which translates into the distance as we assume a constant flight speed. Also, for the scenario to be realistic, the trajectory should not pass through buildings. In terms of safety, less maneuvering in a UAV path would be better. The inclusion of more 
parameters would increase the complexity of the algorithm, which would impact its computational time and may not provide the desired outputs for further analysis.

Other important parameters such as flight altitude and flight speed, was decided not to be part of the optimization process but would be analyzed as parameters of the simulation stage.

The above-selected parameters were formed into a cost function which was given to the optimization algorithm for minimization. This formation, which adopts the rationale of [18], is as follows:

$$
\begin{aligned}
& f_{\text {cost }}=f_{\text {dist }}\left(\alpha+\beta f_{\text {obs }}+\gamma f_{\text {head }}\right), \text { is the overall cost funtion to be minimized } \\
& f_{\text {dist }}=\sum_{i=1}^{n-1} d\left(P_{i}, P_{i+1}\right)=\sum_{i=1}^{n-1} \sqrt{\left(x_{i+1}-x_{i}\right)^{2}+\left(y_{i+1}-y_{i}\right)^{2}} \\
& \text { is the Euclidean distance of two consecutive points in the path }
\end{aligned}
$$

$$
f_{\text {obs }}=\left\{\begin{array}{rr}
1, & \text { if the path is not free of obstacles } \\
0, & \text { if the path is free of obstacles }
\end{array}\right.
$$

$f_{\text {head }}=\sum_{i=1}^{n-1}\left|\theta_{i}\right|=\sum_{i=1}^{n-1}\left|\tan ^{-1} \frac{\left(y_{i+1}-y_{i}\right)}{\left(x_{i+1}-x_{i}\right)}\right|$, is the heading angle

$\alpha, \beta, \gamma$ : are weighting factors and $n:$ is the number of handle points of the path

The choice of weighting factors $(\alpha=1, \beta=1000, \gamma=0,1)$ was made after conducting an experiment to decide the most efficient combination of weightings that give more importance to the path being free of obstacles (buildings and low GNSS quality areas) and less to its total distance and smoothness.

The PSO algorithm implementation also consisted of the following parameters that required tuning:

- $n$ : Number of handle points for path computation. The higher the $\mathrm{n}$, the more precise the path would be, but also the algorithm complexity would increase dramatically.

- iter: Number of maximum iterations for the algorithm to be terminated.

- pop: Swarm population size. The higher the population, the more possible it is to find an optimal solution, but also more computational power and time is needed.

After experimenting, the best values of the above parameters were decided to be $n=6$, iter $=200$ and $p o p=60$. The internal PSO parameters, such as inertial weight, damping ratio, personal and global learning coefficient, were left to its default values. The PSO algorithm was executed in MATLAB version R2019b on a PC with Intel Core i7-7700 CPU running @ 3.6 GHz, 16 GB RAM and under 64-bit Windows 10 Enterprise OS.

\section{E. Trajectory Simulation}

As described in Section III.C, the optimization process produces trajectories for both cases, one which ignores GNSS parameters as input and one that includes them. As a result, in the first case the trajectory passes "through" the urban canyon and in the second case it tries to avoid the GNSS challenging environment of the urban canyon, so produces an "around" trajectory.

The optimal trajectory outputs of the previous stage were then simulated in the Cranfield UAV Technology LAB using the setup described in Section III.A.

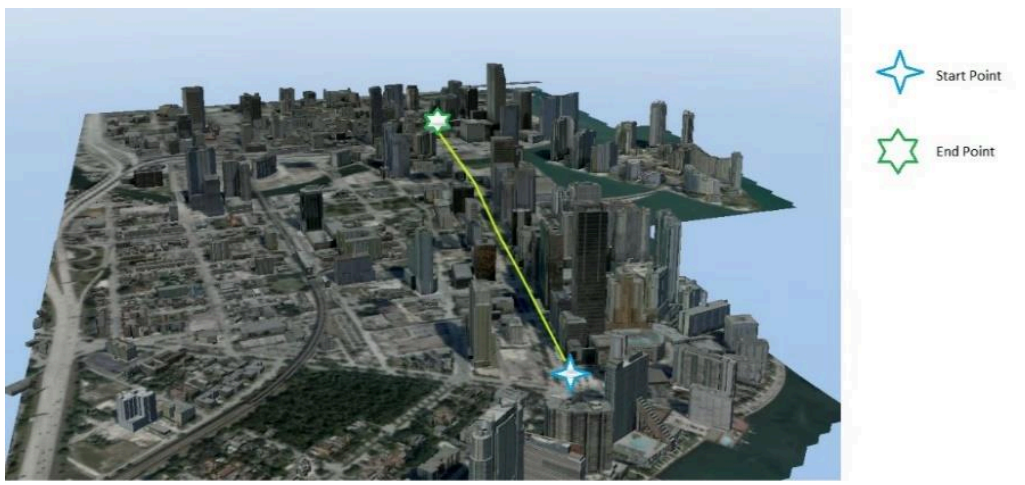

Figure 6 Start and end points of the simulations

All simulations were run at the same Date/Time: $2^{\text {nd }}$ July 2020, 15:00 UTC. In order to assess the impact of the mission requirements when flying "through" the Miami urban canyon and when flying "around", the start and end points of all the simulations were picked as seen in Figure 6, in the beginning, and at the end of the urban canyon. Also, all trajectories were simulated using different altitude and flight speed values:

- Flight altitude: $10 \mathrm{~m}, 30 \mathrm{~m}$ and $100 \mathrm{~m}$ above ground level.

- Flight speed: $3 \mathrm{~m} / \mathrm{s}$ and $10 \mathrm{~m} / \mathrm{s}$. 
The selection for the altitude values was made in order to investigate how the GNSS signal quality is affected when flying at a low, medium and high altitudes, both through and around the urban canyon. In the same context, the speed selection was made in order to investigate what the impact of flying at low and high speeds could be.

Another parameter that should be selected by the SimGEN software was the GNSS constellation. It was decided that only the GPS constellation case would be investigated for this research. The investigation of the impact of GNSS quality on the path planning optimization considering multiple GNSS constellations is planned to be covered in future works.

The reference trajectory, which was considered the ground truth of the UAV path for each mission scenario, was simulated by using only the SimGEN software, by taking into account only the waypoints created, the date/time, the flight altitude and the flight speed.

In total, 60 simulations were performed (5 multipaths x 3 altitudes $x 2$ speeds $x 2$ paths). The summary of all the parameters that were changing in each simulation can be seen in Table 1.

Table 1 Simulation parameters summary

\begin{tabular}{|c|c|c|c|c|}
\hline Parameter & Multipath & Altitude & Speed & Path \\
\hline \multirow{5}{*}{ Values } & 0 multipath (only direct beam) & $10 \mathrm{~m}$ & $3 \mathrm{~m} / \mathrm{s}$ & Through \\
& 1 multipath - 1 reflection & $30 \mathrm{~m}$ & $10 \mathrm{~m} / \mathrm{s}$ & Around \\
& 1 multipath - 2 reflections & $100 \mathrm{~m}$ & & \\
& 2 multipaths - 1 reflection & & & \\
& 2 multipaths - 2 reflections & & & \\
\hline
\end{tabular}

\section{IV.Analysis and Discussion}

In the first case where the GNSS parameters are not included in the optimization process, the PSO calculates the optimal path by trying to minimize the distance between the start and end points and minimize the heading angle change rate. The result is that the optimal path passes "through" the urban canyon. Whereas, in the second case, where GNSS observations are taken into account in the PSO, the result is that the urban canyon where GNSS quality is degraded, is being avoided and the optimal UAV path leads "around" it. Illustration of simulated trajectories can be seen in Figure 7.
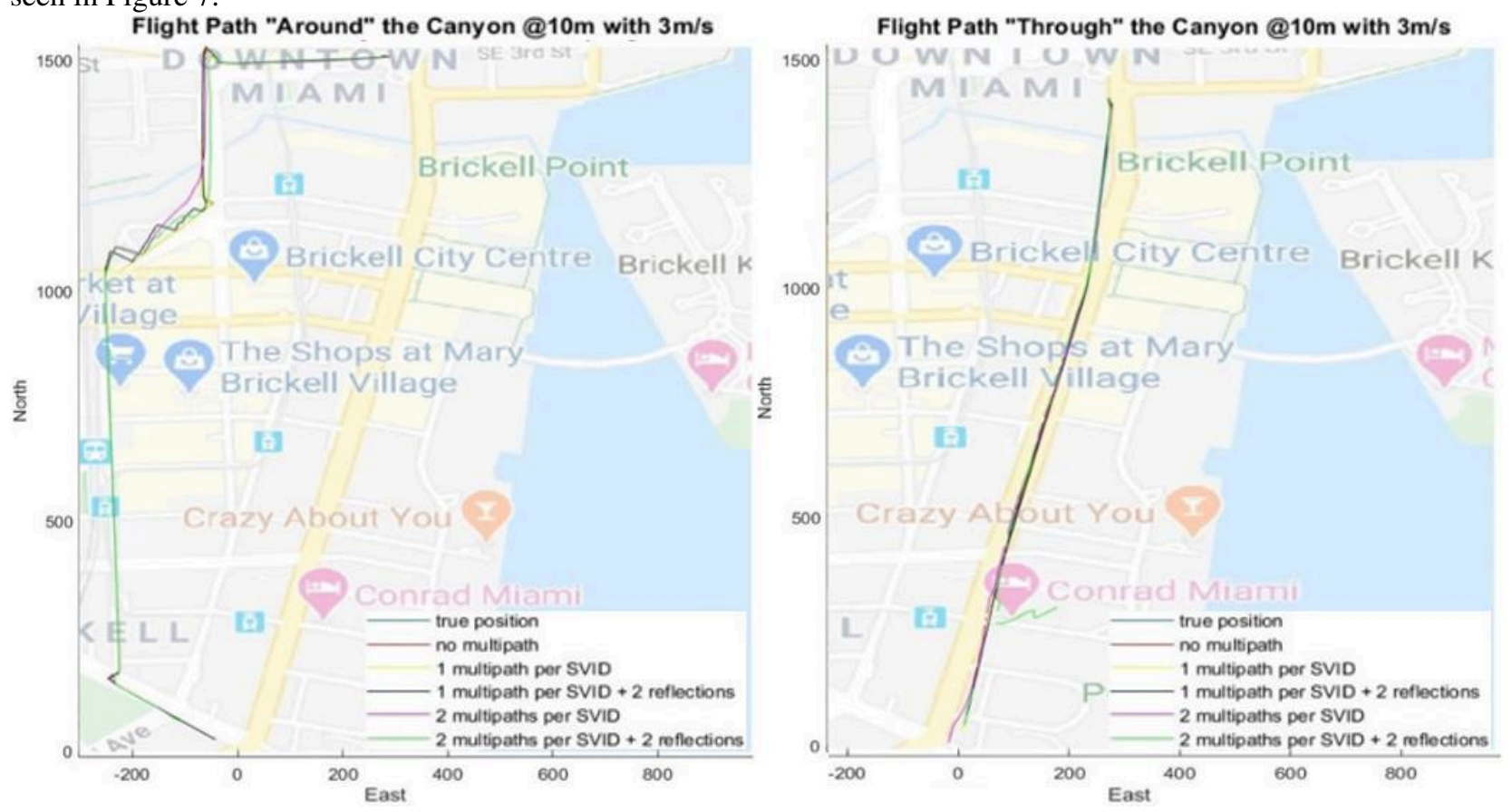

Figure 7 Simulated trajectories around and through the urban canyon. 
In order to validate the findings of the optimization procedure, all the optimal paths were simulated as described in Section III. The optimal paths for the "through" the canyon and the "around" the canyon case were simulated 30 times each one, according to the information in Table 1, so there was a total of 60 simulations. For each simulation, the mission failure cases were calculated, as summarized in Table 2. For the simulation analysis, two cases of mission failures are considered. The first one, when there are missing GNSS data (NVS < 4) for more than Time-to-Alert (TTA) (10 s) and the second one, when the positioning error of the UAV path compared to the reference trajectory is more than the Horizontal Alert Limit (HAL) $(10 \mathrm{~m})$ for more than TTA (10 s).

Table 2 Mission Failure Definition

\begin{tabular}{|c|c|}
\hline MISSION FAILURE CASE & CONDITION \\
\hline MF_A & $(\mathrm{NVS}<4)$ for (Time $>$ TTA $=10 \mathrm{~s})$ \\
\hline MF_B & $($ PosErr $>$ HAL $=10 \mathrm{~m})$ for $($ Time $>$ TTA $=10 \mathrm{~s})$ \\
\hline
\end{tabular}

In this context, from the GNSS integrity point of view, a HAL regarding positioning error was decided to be defined at the boundary between flight and contingency volume, as described in Section II. The value of the HAL was decided to be $10 \mathrm{~m}$. For this selection, the average street width of the Miami area (urban canyon and surroundings), which is $30 \mathrm{~m}$, was taken into consideration. TTA value is selected as $10 \mathrm{~s}$, which is defined for safety-critical applications in [19], as our application is also considered safety-critical. This selection gives the UAV sufficient time to recover from its first entry into the contingency volume. The selected TTA value is also sufficient from the GNSS availability point of view, e.g. in the case where GNSS data are missing during a simulation, i.e. when the number of visible satellites for the GNSS receiver has fallen below 4, such as for GPS no Fix case.

For the Mission Success Rate (MSR) definition of each mission scenario (fixed altitude and fixed speed), the number of Mission Failures that occur in each of the 5 multipath cases, as described in Table 1, are counted for each Mission Failure case (Table 2) separately. The corresponding Mission Success Rates (MSR_A and MSR_B) as well as the overall MSR are defined as follows:

$$
\begin{aligned}
& M S R_{A}=\left(1-\frac{N_{M F_{-} A}}{N_{S I M}}\right) * 100 \% \\
& M S R_{B}=\left(1-\frac{N_{M F_{-} B}}{N_{\text {SIM }}}\right) * 100 \%
\end{aligned}
$$

$N_{M F_{-} A}:$ Mean number of mission failures per scenario

$N_{M F_{-} B}$ : Mean number of mission failures per scenario

$N_{S I M}$ :Total number of simulations per scenario

$$
M S R=\frac{\left(M S R_{A}+M S R_{B}\right)}{2}
$$

The overall MSR value of a mission scenario is the indication of the probability that this scenario is entirely successful, i.e. without any failures as defined in Table 2. The closer the MSR to $100 \%$, the more probable it is to be without failure.

Accordingly, the mission success rates were computed as in equations (5) to (7). The calculated values for MF_A,

\begin{tabular}{|c|c|c|c|c|c|c|c|}
\hline \multicolumn{3}{|c|}{ CASE } & $\begin{array}{r}\text { Overall } \\
\text { failed A }\end{array}$ & $\begin{array}{r}\text { Overall } \\
\text { failed B }\end{array}$ & MSR_A & MSR_B & MSR \\
\hline \multirow{6}{*}{$3 \mathrm{~m} / \mathrm{s}$} & \multirow{2}{*}{$10 \mathrm{~m}$} & Through & 2 & 4 & 0.6 & 0.2 & 0.4 \\
\hline & & Around & 1 & 3 & 0.8 & 0.4 & 0.6 \\
\hline & \multirow{2}{*}{$30 \mathrm{~m}$} & Through & 0 & 4 & 1 & 0.2 & 0.6 \\
\hline & & Around & 0 & 3 & 1 & 0.4 & 0.7 \\
\hline & \multirow{2}{*}{$100 \mathrm{~m}$} & Through & 0 & 0 & 1 & 1 & 1 \\
\hline & & Around & 0 & 2 & 1 & 0.6 & 0.8 \\
\hline
\end{tabular}
MF_A, MSR_A, MSR_B and MSR are summarized in Table 3.

Table 3 Summary of simulation results. 


\begin{tabular}{|l|c|c|c|c|c|c|c|}
\hline \multirow{3}{*}{$10 \mathrm{~m} / \mathrm{s}$} & \multirow{3}{*}{$10 \mathrm{~m}$} & Through & 5 & 3 & 0 & 0.4 & 0.2 \\
\cline { 3 - 8 } & \multirow{3}{*}{$30 \mathrm{~m}$} & Around & 2 & 1 & 0.6 & 0.8 & 0.7 \\
\cline { 3 - 8 } & & Through & 5 & 1 & 0 & 0.8 & 0.4 \\
\cline { 2 - 7 } & \multirow{2}{*}{$100 \mathrm{~m}$} & Around & 2 & 2 & 0.6 & 0.6 & 0.6 \\
\cline { 3 - 8 } & & Through & 4 & 2 & 0.2 & 0.6 & 0.4 \\
\cline { 3 - 8 } & & Around & 1 & 1 & 0.8 & 0.8 & 0.8 \\
\hline
\end{tabular}

From the simulation results, it can be observed that the overall MSR is improved in 5 out of 6 cases (83.3\%) when the "around" path of the path planning optimization process is followed instead of the "through" path. The only case where it is not improved is at high altitude $(100 \mathrm{~m})$ and low speed $(3 \mathrm{~m} / \mathrm{s})$, in which case the MSR is very high in both cases (100\% "through" and $80 \%$ "around"). This is a good indication that the inclusion of GNSS parameters in the path planning process can improve the probability of a mission being successful. This can also be identified as increasing the mission safety factor.

Moreover, when flying "through" the canyon at low speed $(3 \mathrm{~m} / \mathrm{s})$, as the altitude increases the corresponding MSR increases as well and reaches $100 \%$ at $100 \mathrm{~m}$ altitude. This is an expected finding, as in higher altitudes, the urban environment is less dense, and it does not affect the GNSS signal quality as much as in lower altitudes.

Furthermore, when flying at high speed $(10 \mathrm{~m} / \mathrm{s})$, the improvement of MSR due to path planning in all three altitudes is much bigger compared to low speed $(3 \mathrm{~m} / \mathrm{s})$, as shown in Figure 8 MSR when flying at constant speeds.. This is mainly because of the degradation of GPS availability at high speed when flying through the canyon, as shown in Figure 9. The GPS availability (NVS < 4) is the main contributor for mission failures when flying through the canyon at high speeds regardless of the flight altitude. Also, at high speed $(10 \mathrm{~m} / \mathrm{s})$, flying the optimal path "around" the canyon has an impressive positive impact of $60 \%$ in the MSR_A at every altitude when only MSR_A is considered.
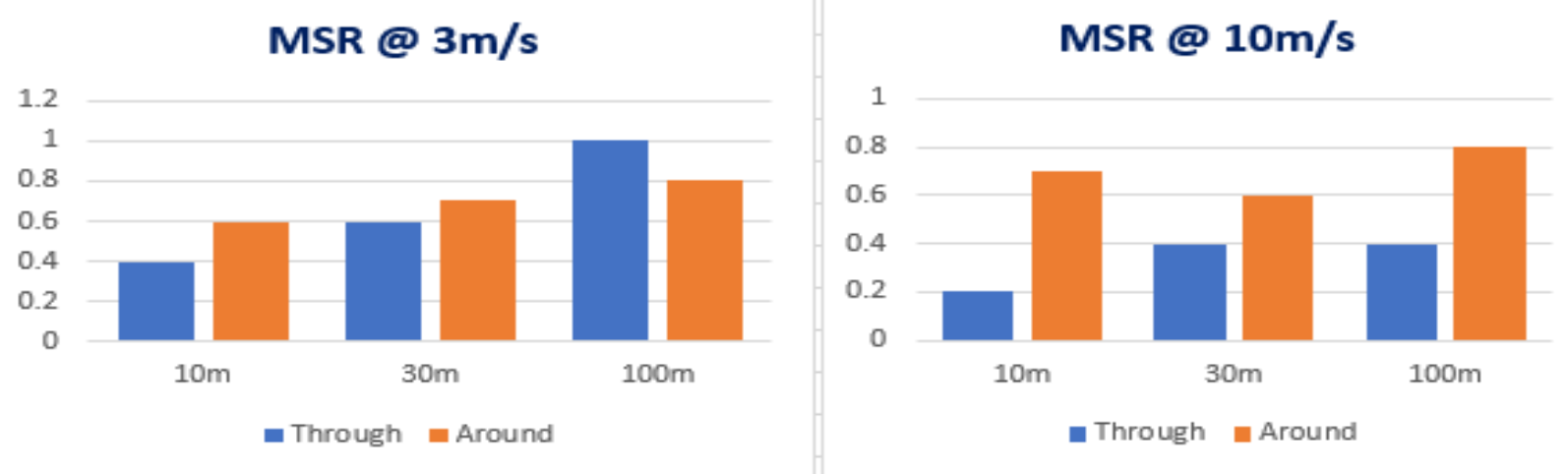

Figure 8 MSR when flying at constant speeds.

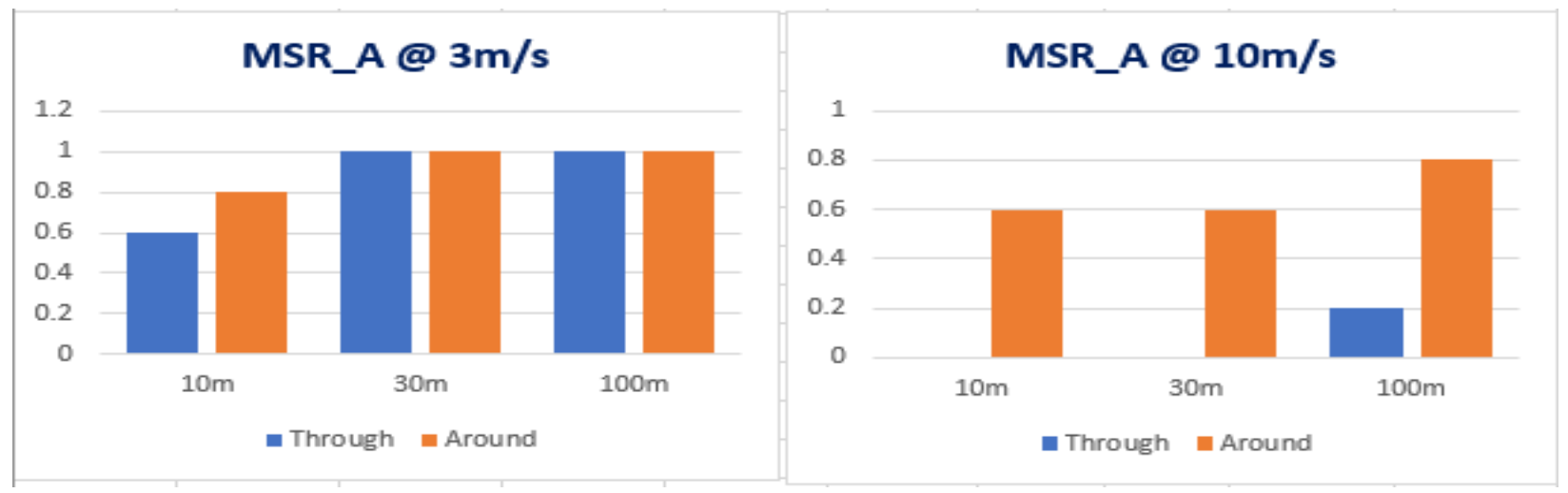

Figure 9 MSR due to NVS $<4$ at constant speeds.

In contrast, at lower speeds ( $3 \mathrm{~m} / \mathrm{s})$, GPS availability seems not to be an issue when flying over $30 \mathrm{~m}$. At $10 \mathrm{~m}$, when flying the optimal "around" path, there is an increase of $20 \%$ in the corresponding MSR. So, when a flight 
through the urban canyon is mandatory, the results show that a low-speed flight would significantly increase the GPS availability at all altitudes. This may be caused by the intense degradation of signal acquisition. Under low visibility conditions, slow movement speed provides more time to receiver for position estimations.

Considering the mission failures due to positioning error in Figure 10 it can be seen that the path planning process improves the MSR_B only at the low flight altitude $(10 \mathrm{~m})$ and the medium $(30 \mathrm{~m})$ when flying at low speed $(3 \mathrm{~m} / \mathrm{s})$. Also, in nearly all cases, the MSR_B performance improves when flying at high speed $(10 \mathrm{~m} / \mathrm{s})$, compared to low speed $(3 \mathrm{~m} / \mathrm{s})$, regardless of the flight altitude in both "through" and "around" the canyon cases.

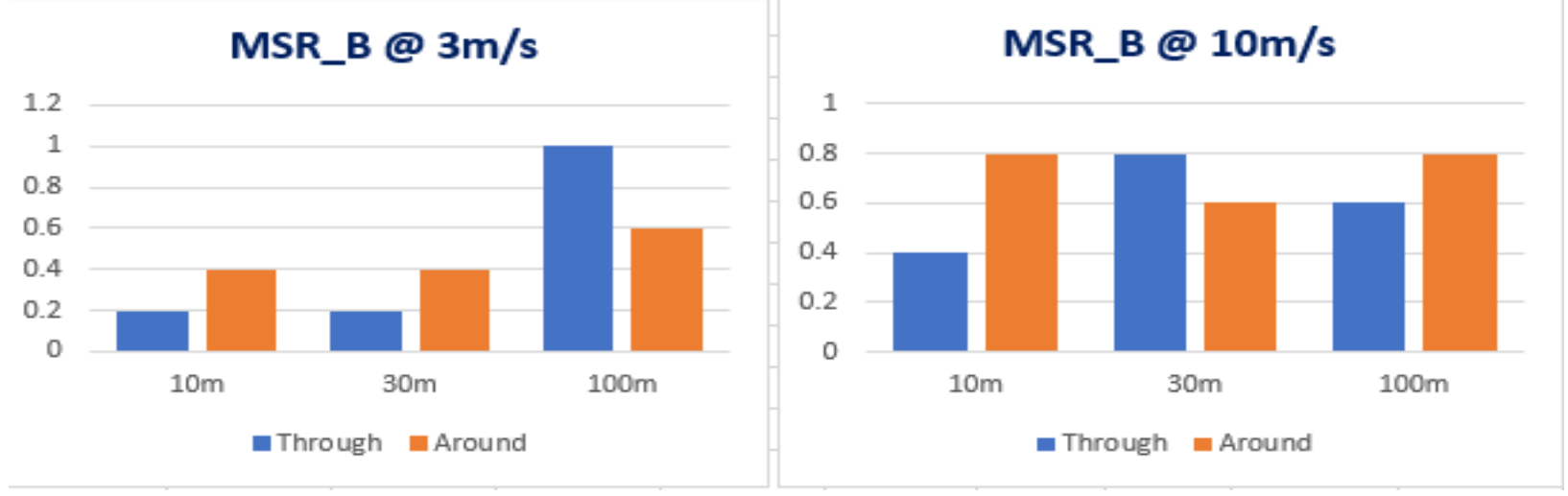

Figure 10 MSR due to positioning error at constant flight speeds.

Finally, from Figure 11, it is clear that an increase in speed during a flight "through" the canyon would have a negative impact in the overall MSR at all altitudes. This is mostly because of the degraded GPS availability at high speed, as explained above. For the path "around" the canyon, the increase of the flight speed has only a small impact in the overall MSR.

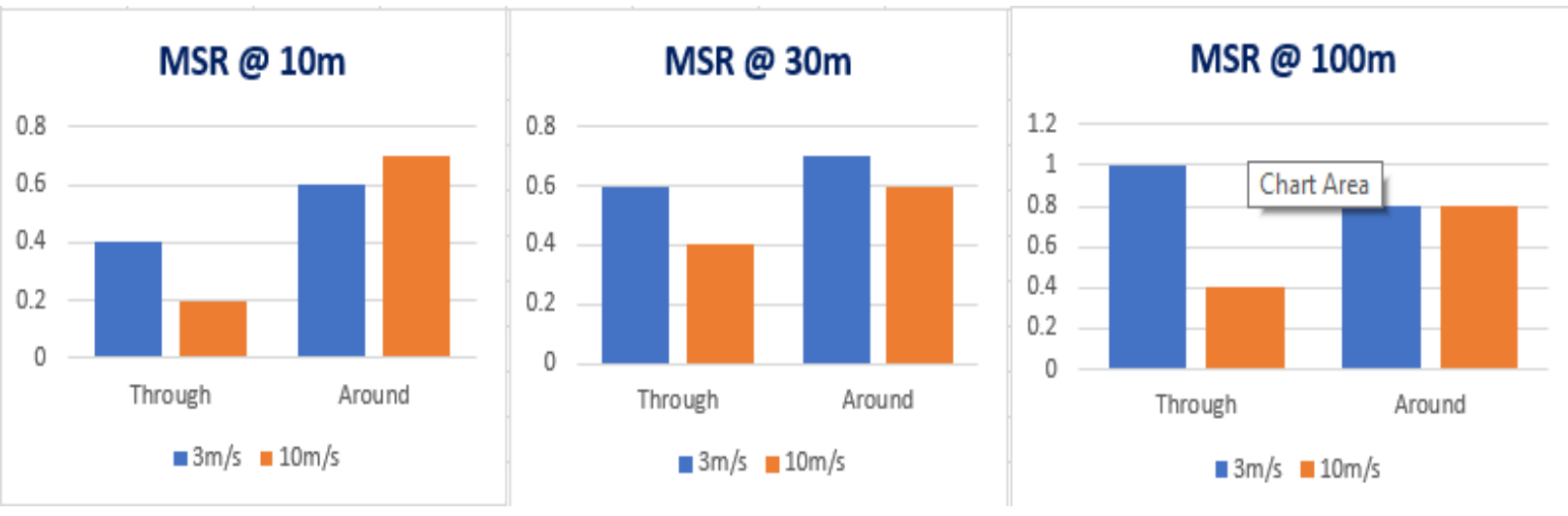

Figure 11 MSR at constant flight altitudes.

To summarize the above experimental findings, the proposed in this paper method of including GPS observations in the path planning optimization process has a positive impact on the mission success rate in the majority of the cases (83\%). It was found that the mission success rate depends on flight altitude selection: the lower the UAV flight, the worse the MSR in general. The proposed method increases the MSR at low altitudes by $20 \%$. At higher altitudes, the MSR values are better, and the improvement due to the proposed path planning procedure is lower, but still possible.

When the flight speed is concerned, it can be deduced that a low flight speed gives a better performance in MSR when flying through the urban canyon. In contrast, when flying around the canyon due to path planning, the average performance seems not to be affected by the change in flight speed. As far as mission failure metrics are concerned, there seems to be a relationship between these metrics and the flight speed. More particular, when flying at low speed, the mission failures due to positioning error exceeding the HAL set is dominant. The opposite occurs when flying at high speeds, where the mission failures due to unavailable GPS (NVS $<4)$ are the majority.

It can be seen that variation in multipath severity, represented in this work through number of multipath beams and reflections variation, leads to an increase in the number of mission failures. However, this trend is observed only at low flight speeds. When flying at higher speeds, the number of mission failures and the multipath parameters do not indicate any increase in mission failures. 
In general, according to the above findings, better results are obtained when flying at low speed $(3 \mathrm{~m} / \mathrm{s})$, compared to the high speed, where the results do not follow well a designed trajectory. The characteristic example of such behavior represents poor GPS availability (NVS < 4) at $100 \mathrm{~m}$ altitude. Therefore, if there is a possibility for a flight speed selection during path planning, the preference should be given to a low speed.

We have also found that the positioning error metric of mission failure is better suited for the path planning process as it describes the variance in the results from case to case. The other metric used, the number of visible satellites, is less efficient, in particular at low speeds and altitude of $30 \mathrm{~m}$ and $100 \mathrm{~m}$, where the GPS availability is $100 \%$ for all cases. Therefore, if someone wants to select a single metric to account for GNSS performance in path planning, the results suggest this to be the positioning error.

\section{Conclusion}

This paper proposes the inclusion of GNSS parameters in the UAV path planning optimization process to maximize its mission success rate. GNSS availability and integrity measures (Number of Visible Satellite and Positioning Errors, represented by DOP coefficients) are used as input in the path planning algorithm (based on PSO) to produce flight paths, which are validated using hardware GNSS simulator and realistic multipath and 3D city model of Miami. The experimental results suggest that if the proposed method is followed, the overall mission success rate can be increased in the majority of the cases (83\%). Additionally, the path planning process has better results (the increase in MSR is bigger) when flying at low altitude $(10 \mathrm{~m})$. In future it is planned to investigate the performance of the method if multiple GNSS constellations would be used instead of the GPS-only case.

\section{References}

[1] Coelho, B. N., Coelho, V. N., Coelho, I. M., Ochi, L. S., Haghnazar K., R., Zuidema, D., Lima, M. S. F., and da Costa, A. R. “A Multi-Objective Green UAV Routing Problem.” Computers and Operations Research, Vol. 88, 2017, pp. $306-315$. https://doi.org/10.1016/j.cor.2017.04.011.

[2] Jeong, H. Y., Song, B. D., and Lee, S. “Truck-Drone Hybrid Delivery Routing: Payload-Energy Dependency and No-Fly Zones." International Journal of Production Economics, Vol. 214, 2019, pp. $220-233$. https://doi.org/10.1016/j.ijpe.2019.01.010.

[3] Rosenow, J., Förster, S., Lindner, M., and Fricke, H. Impact of Multi-Critica Optimized Trajectories on European Air Traffic Density, Efficiency and the Environment. 2017.

[4] Civil Aviation Authority. CAP1915, Unmanned Aircraft Systems BVLOS Operations in Support of the COVID-19 Response-Requirements, Guidance \& Policy. 2020.

[5] Aggarwal, S., and Kumar, N. Path Planning Techniques for Unmanned Aerial Vehicles: A Review, Solutions, and Challenges. Computer Communications. Volume 149, 270-299.

[6] Kennedy, J., Eberhart, R., and Gov, B. Particle Swarm Optimization. 1995.

[7] ICAO, I. C. A. O. Aeronautical Telecommunications Annex 10 to the Convention on International Civil Aviation Standards and Recommended Practices and Procedures for Air Navigation Services Volume II Communication Procedures Including Those with PANS Status. 2006.

[8] Struempfel, C., Schuster, E., Huschbeck, S., Berth, C., and Uijt De Haag, M. Assured Multi-Mode Navigation for Urban Operations of Small UAS. 2020.

[9] Zhang, G., and Hsu, L. T. “A New Path Planning Algorithm Using a GNSS Localization Error Map for UAVs in an Urban Area." Journal of Intelligent and Robotic Systems: Theory and Applications, Vol. 94, No. 1, 2019, pp. $219-235$. https://doi.org/10.1007/s10846-018-0894-5.

[10] Causa, F., Fasano, G., and Grassi, M. GNSS-Aware Path Planning for UAV Swarm in Complex Environments. 2019.

[11] Spirent. GSS7000 Series GNSS Constellation Simulator, MS7000 Datasheet with Product Specification, Crawley, UK, 2020.

[12] Spirent, P. "SimGEN ® Software Suite for Spirent GNSS Constellation Simulation Systems, MS3008 Datasheet with Product Specification, Crawley, UK, 2019."

[13] Spirent, P. Sim3D Software, MS3105 Datasheet with Product Specification, Crawley, UK, 2019

[14] Ublox. "Neo-M8P." UBX-15031086-R07 Datasheet with Product Information, 2020.

[15] HMG. "The Air Navigation Order 2009." National Archives (legislation.giv.uk), Vol. 2, 2009, pp. 1-2.

[16] National Aeronautics and Space Administration (NASA). "Unmanned Aircraft System (UAS) Traffic Management (UTM)." Enabling Civilian Low-altitude Airspace and Unmanned Aircraft System Operations, 2019, p. https://utm.arc.nasa.gov/index.shtml.

[17] Isik, O. K., Hong, J., Petrunin, I., and Tsourdos, A. "Integrity Analysis for GPS-Based Navigation of UAVs in Urban Environment." 2020, pp. 1-20.

[18] Fetanat, M., Haghzad, S., and Shouraki, S. B. Optimization of Dynamic Mobile Robot Path Planning Based on Evolutionary Methods. 2015.

[19] European GNSS Agency. "Report on the Performance and Level of Integrity for Safety and Liability Critical MultiApplications." No. May, 2015, p. 44. 
2021-01-04

\section{UAV path planning optimization based on GNSS quality and mission requirements}

Nanos, Nikolaos

AIAA

Nanos N, Kagan Isik O, Verdeguer Moreno R, et al., (2021) UAV path planning optimization based on GNSS quality and mission requirements. In: AIAA SciTech Forum 2021, Virtual Event, 19-21 January 2021

https://doi.org/10.2514/6.2021-0710

Downloaded from Cranfield Library Services E-Repository 